\title{
Real-Time Magnetic Resonance Imaging of Temporomandibular Joint Dynamics
}

\author{
Shuo Zhang*,1, Nikolaus Gersdorff ${ }^{2}$ and Jens Frahm ${ }^{1}$ \\ ${ }^{I}$ Biomedizinische NMR Forschungs GmbH am Max-Planck-Institut für biophysikalische Chemie, Am Fassberg 11, \\ 37070 Göttingen, Germany; ${ }^{2}$ Department of Prosthodontics, Georg-August-University Göttingen, 37075 Göttingen, \\ Germany
}

\begin{abstract}
This study evaluated the use of a novel real-time MRI technique based on fast low angle shot (FLASH) MRI with radial encoding, gridding reconstruction, and sliding window for the assessment of temporomandibular joint (TMJ) dynamics in a cohort of 30 young volunteers without prior diagnosis of TMJ pathology. High-resolution images $(0.75 \times$ $0.75 \mathrm{~mm}^{2}, 5 \mathrm{~mm}$ section thickness) were obtained at 3 frames per second for active jaw movements without adjunctive devices. Real-time movies were evaluated with respect to image artefacts, anatomical visibility, diagnostic confidence, and TMJ function. During the entire opening and closing of the mouth, the relative positions of the mandibular condyle and articular disc were well depicted. Mean scores were $1.01 \pm 0.65$ for motion artefacts (scale: $0=$ no to $3=$ severe artefacts) and $2.03 \pm 0.71$ for anatomical detectability (scale: $1=$ excellent to $5=$ not visible). High inter-observer agreements were found for assessments of mandibular condyle movements $(\kappa=0.83)$ and articular disc displacements $(\kappa$ $=0.91$ ). In latter cases, the point of disc reduction could precisely be identified. In conclusion, the proposed real-time MRI method offers robust access to TMJ dynamics with good image quality.
\end{abstract}

Keywords: Temporomandibular joint, magnetic resonance, MRI, dynamic MRI, joint.

\section{INTRODUCTION}

Diagnoses of temporomandibular joint (TMJ) disorders mainly involve imaging of the internal derangement, which often refers to an abnormal position of the articular disc relative to the mandibular condyle and articular eminence, i.e. an abnormal disc-condyle relationship and respective movements. Because clinical examinations alone cannot fully assess these abnormalities [1], magnetic resonance imaging (MRI) has widely been accepted for the evaluation of TMJ disorders [2]. This is largely due to its noninvasiveness, the access to arbitrary section orientations, and the native soft-tissue contrast. However, dynamic assessments of joint function remain a serious challenge. Early attempts by cine MRI relied on pseudo-dynamic recordings and employed a bite block or similar adjunctive device to control the mouth opening position for sequential step-by-step acquisitions [3-5]. Apart from the long measuring times and cumbersome examinations, the retrospective combination of images that represent static positions is physiologically not equivalent to the situation encountered during active jaw movement [6].

Alternatively, a number of fast pulse sequences have been proposed for dynamic MRI [7-13], but so far no generally accepted approach of sufficient quality has evolved. Typical problems comprise susceptibility-induced

*Address correspondence to this author at the Biomedizinische NMR Forschungs GmbH am Max-Planck-Institut für biophysikalische Chemie, 37070 Göttingen, Germany; Tel: +49-551-201-1735;

E-mail: szhang1@gwdg.de signal losses (e.g., for echo-planar imaging), sensitivity to off-resonance effects (e.g., balanced steady-state free precession sequences), or limited spatial resolution.

The aim of this contribution is to introduce a recently developed real-time MRI technique that provides a promising solution for the aforementioned problems. The approach combines fast low-angle shot (FLASH) gradientecho MRI pulse sequences with a radial data encoding scheme and a sliding window reconstruction using gridding [14]. In this article, we describe the first application of this novel technique to a group of young and previously undiagnosed subjects who presented with both normal and abnormal TMJ function.

\section{MATERIALS \& METHODS}

\section{Subjects}

Thirty volunteers ( 8 men, 22 women; mean age $24.5 \pm$ 2.9 years [standard deviation]; age range $20-31$ years) without prior diagnosis of TMJ disorder were recruited among the students of the local University. The whole study was approved by the institutional review board and all participants gave written informed consent before each MRI examination. Before MRI all subjects were physically examined by one author with 5 years of experience specialized in clinical examinations according to the guidelines of Axis I RDC/TMD [15].

\section{Real-Time MRI}

All studies were conducted at 3 Tesla using a commercially available MRI system (Tim Trio, Siemens Healthcare, Erlangen, Germany). Subjects were examined in 
a supine position with the use of a bilateral $2 \times 4$ array coil (NORAS MRI products, Hoechberg, Germany) offering two independent and adjustable 4-element coils for the TMJ on both sides. The coils were positioned parallel to the Frankfurt horizontal plane (Fig. 1A) and imaging sections were chosen in an oblique sagittal orientation perpendicular to the long axis of the condylar head (Figs. 1B and 1C).

Prior to dynamic MRI, static multi-slice scout images in the closed-mouth position were obtained using the same sequence as applied for real-time MRI. For dynamic imaging, we employed a refocused radial FLASH MRI sequence with an in-phase condition for overlapping water and fat signals, because it offers the best visibility of joint structures, as demonstrated in Fig. (2). The imaging parameters were: repetition time $\mathrm{TR}=4.3 \mathrm{~ms}$, echo time $\mathrm{TE}$ $=2.2 \mathrm{~ms}$, and flip angle $20^{\circ}$. In all cases, the field of view and in-plane resolution were $192 \times 192 \mathrm{~mm}^{2}$ and $0.75 \times 0.75$ $\mathrm{mm}^{2}$, respectively. Three sections with a thickness of $5 \mathrm{~mm}$ each contiguously covered the medial, central, and lateral part of the condylar head. Individual images were obtained from 385 spokes with a radial encoding scheme that involved 5 interleaved turns each covering $360^{\circ}$ of k-space with 77 spokes [14]. The total image acquisition time was $1667 \mathrm{~ms}$, while image updates for sliding window reconstructions were obtained after completion of each of the 5 turns, which yielded a temporal resolution of 3 frames per second.

Voluntary jaw movements were instructed by a video and the use of a projection setup (beamer, lens, screen, mirror) as employed for MRI studies of human brain function (Fig. 1A). Each dynamic recording lasted for about $50 \mathrm{~s}$ and included one full cycle of maximum opening and closing of the mouth with a slow continuous movement. Altogether, the total MRI examination time was less than 8 min per subject.

\section{Image Evaluation}

All real-time MRI data sets (movies) were quantitatively evaluated by independent observers blinded to the subject data and the clinical examination results. The subject order was randomized, but images from the same subject were reviewed at the same time according to diagnostic practice.

The presence of artefacts was assessed for 180 movies (6 movies per subject, 30 subjects) by three MRI physicists with 30,25 , and 5 years of experience, respectively. The rating was based on a modified four-point scale [11], with a score of 0 indicating no artefacts; a score of 1 , mild artefacts at abrupt movement; a score of 2 , strong artefacts at abrupt movement; and a score of 3 , artefacts throughout the entire movement.

The detectability of the main anatomical structures and the ability to characterize the (patho-) physiological function of the TMJ, in particular with respect to a potential displacement of the articular disc, were evaluated by three clinicians with 20,5, and 4 years of experience in craniomandibular disorders, respectively. The visibility of anatomical structures was rated on a five-point scale, with a score of 1 indicating excellent visibility with very high confidence in diagnostic content; a score of 2 , good visibility with high confidence in diagnostic content; a score of 3, visible with confidence in diagnostic content; a score of 4 , poor visibility without confidence in diagnostic content; and

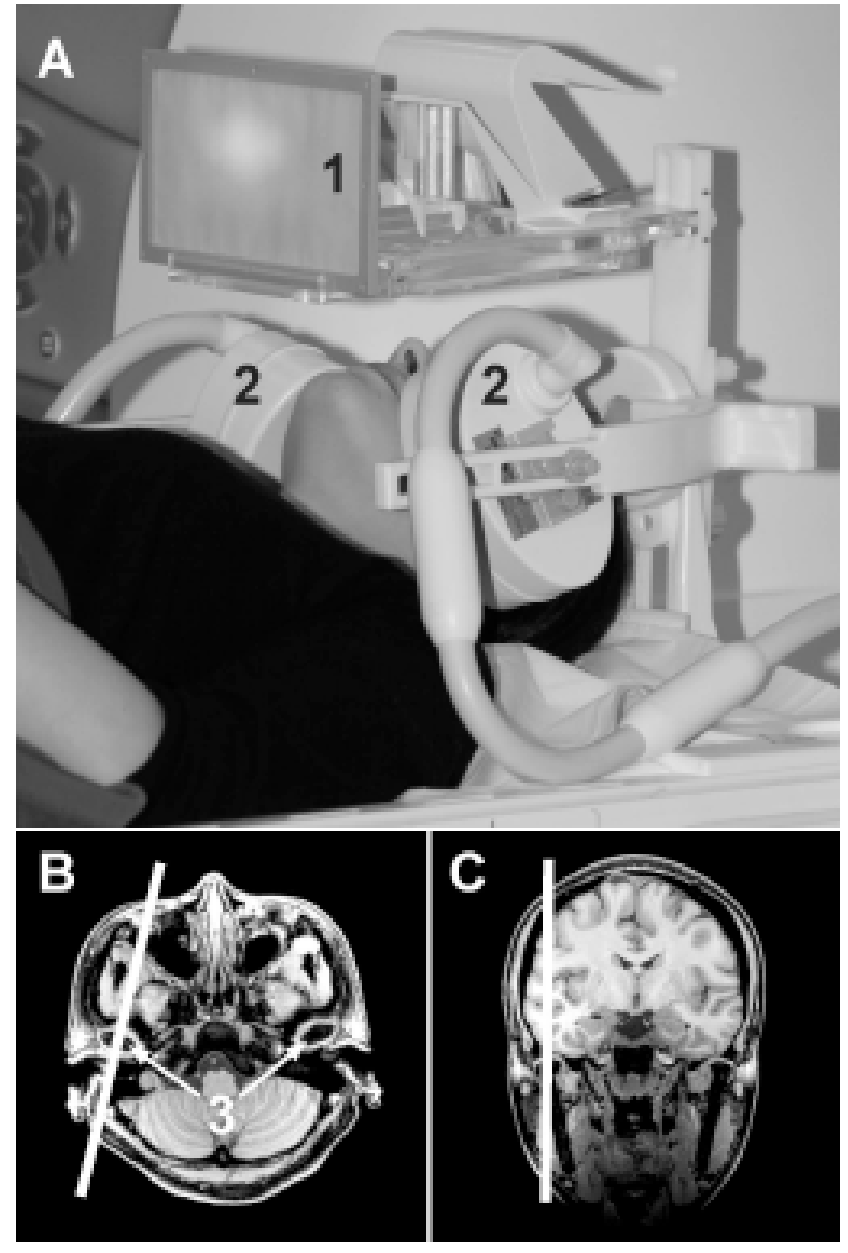

Fig. (1). Setup for real-time MRI of the TMJ. (A) Subjects viewed an instruction video projected onto a screen (1). They were studied in a supine position with a bilateral array coil (2) parallel to the Frankfurt horizontal plane on both sides. (B, C) Real-time MRI was performed in an oblique sagittal orientation perpendicular to the long axis of the condylar head (3).

a score of 5, no visibility and not diagnostic. A rating of 1 to 3 was regarded as positive for detection [11,12].

The disc displacement of each TMJ was characterized as normal, anterior disc displacement with reduction (ADDR), anterior disc displacement without reduction (ADDNR), posterior disc displacement, lateral disc displacement, and medial disc displacement [16]. Condyle movements were classified as normal (with respect to range and continuity), limited (range), and discontinuous [11].

The group-averaged scores for image artefacts and anatomical visibility were given as mean \pm standard deviation. In addition, inter-observer variability was determined by using multi-rater kappa values [17] with the following definitions: $\kappa<0.21$, poor agreement; $\kappa=0.21-$ 0.40 , fair agreement; $\kappa=0.41-0.60$, moderate agreement; $\kappa$ $=0.61-0.80$, substantial agreement; and $\kappa=0.81-1.00$, almost perfect agreement.

\section{RESULTS}

Real-time MRI of the TMJ was not only feasible in all subjects, but also robust in terms of practical performance 


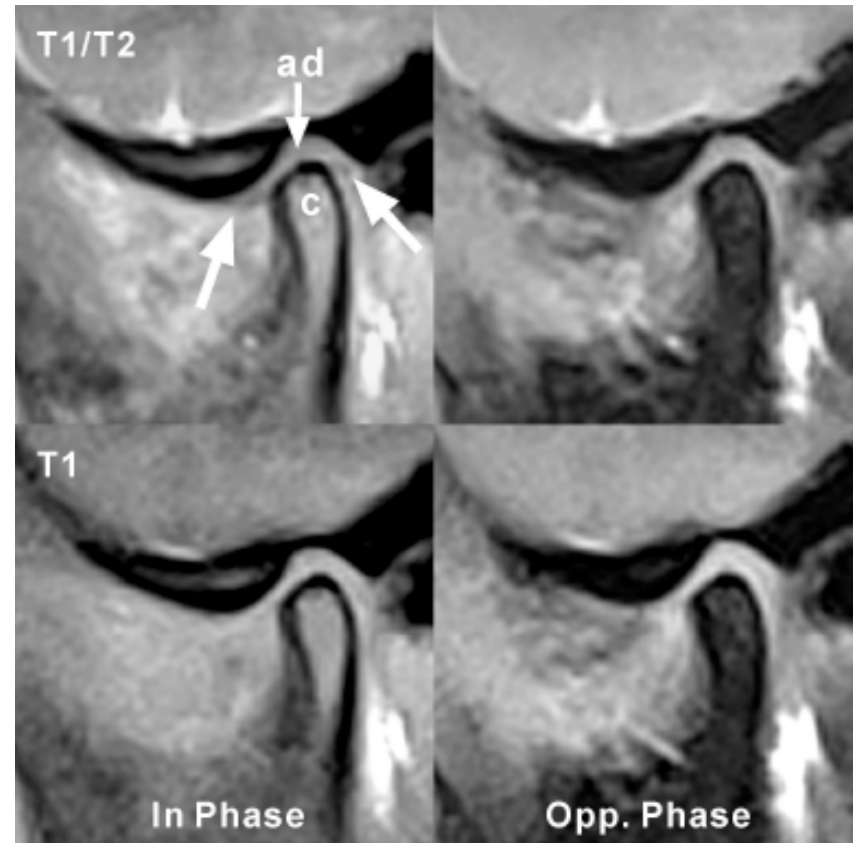

Fig. (2). Real-time MRI of the TMJ at different contrasts (single subject, closed-mouth position). (Top) T1/T2 contrast using refocused radial FLASH vs (bottom) T1 contrast using spoiled radial FLASH for both (left) an in-phase condition (TE $=2.2 \mathrm{~ms}$ ) and (right) an opposed-phase condition (TE $=3.8 \mathrm{~ms})$ for overlapping water and fat signals. ad $=$ articular disc, $\mathrm{c}=$ condylar head, arrows $=$ anterior and posterior borders of the articular disc.

and image quality. Only 2 of 180 movies among 30 subjects required a second measurement to ensure maximal opening of the jaw. Fig. (3A) shows a series of images from a realtime MRI movie (Supportive Movie 1) of a subject with normal TMJ function and no motion artefact during the entire movement cycle.

The clinical examination revealed 21 subjects with normal function, 6 subjects with an internal derangement of one TMJ, and 3 subjects with a bilateral disorder. The MRI findings showed diverse pathologies involving the articular disc, mandibular condyle, and bone. It should be noted,

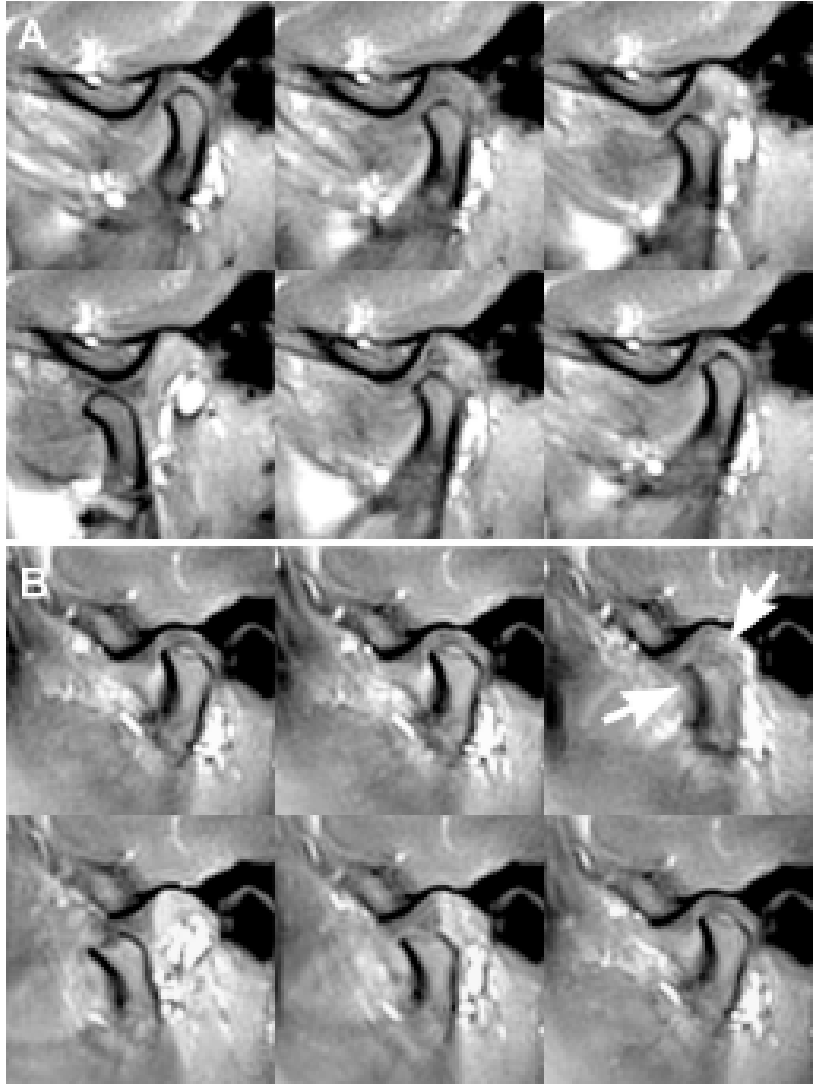

Fig. (3). Real-time MRI of normal TMJ function without (A) and with (B) motion artefact. The images $\left(0.75 \times 0.75 \mathrm{~mm}^{2}, 5 \mathrm{~mm}\right.$ thickness, magnified views) were selected every $11 \mathrm{~s}$ (upper left to lower right) from the corresponding movies (Supportive Movies 1 and 2) of the right TMJ of a 24-year-old male subject and of a 23year-old female subject, respectively. Arrows indicate structural blurring due to an abrupt jaw movement.

however, that the purpose of this pilot study was to test the feasibility of the new real-time MRI method rather than to achieve a comprehensive correlation of MRI findings with clinical data.

Table 1. Rating of Image Artefacts (30 Subjects, 3 Observers)

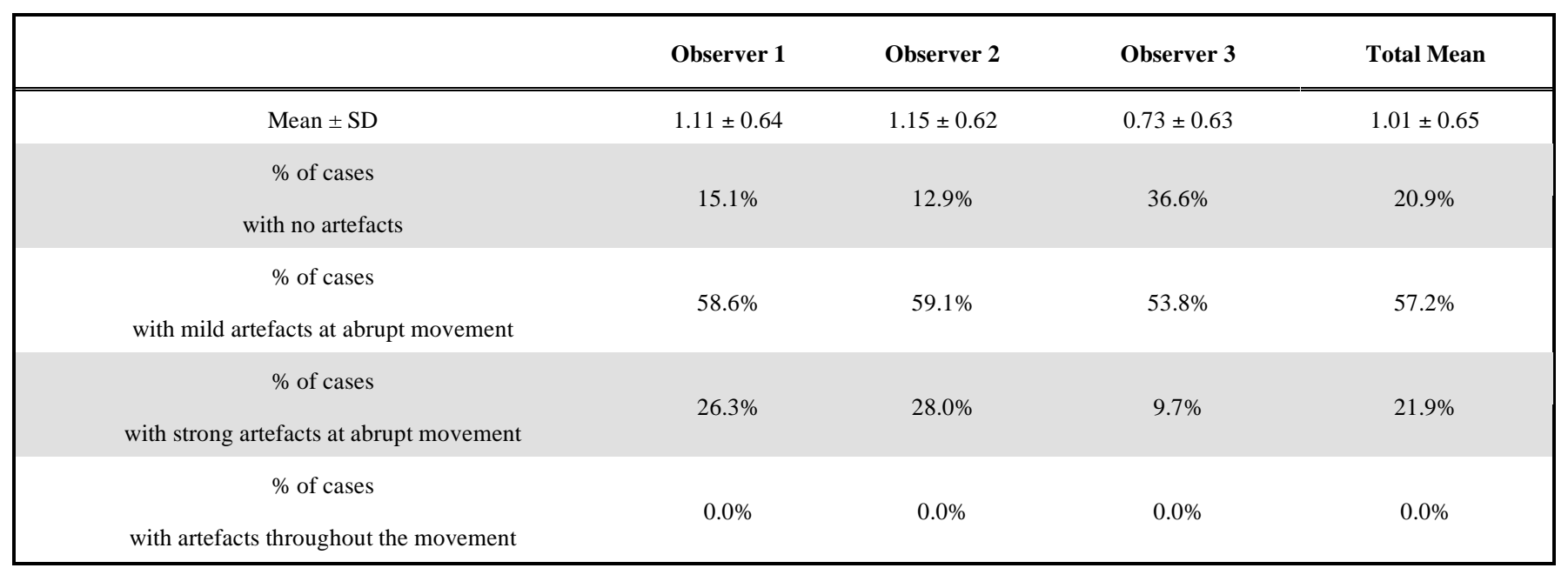


Table 2. Anatomical Visibility and Detection Rate (30 Subjects, 3 Observers)

\begin{tabular}{|c|c|c|c|c|}
\hline & & Mean \pm SD & Detection Rate $(\%)^{\dagger}$ & $\boldsymbol{\kappa}^{\ddagger}$ \\
\hline \multirow{2}{*}{ Anterior border of disc } & Closed-mouth position & $2.53 \pm 0.89$ & $84.9 \%$ & 0.53 \\
\hline & During movement & $2.22 \pm 0.76$ & $97.3 \%$ & 0.89 \\
\hline \multirow{2}{*}{ Posterior border of disc } & Closed-mouth position & $2.89 \pm 0.85$ & $79.0 \%$ & 0.53 \\
\hline & During movement & $2.00 \pm 0.68$ & $98.9 \%$ & 0.96 \\
\hline \multirow[b]{2}{*}{ Disc body } & Closed-mouth position & $2.68 \pm 0.74$ & $91.9 \%$ & 0.76 \\
\hline & Open-mouth position & $1.98 \pm 0.77$ & $97.8 \%$ & 0.98 \\
\hline \multirow[t]{2}{*}{ Condyle head } & Open-mouth position & $1.24 \pm 0.48$ & $99.5 \%$ & 0.98 \\
\hline & During movement & $1.27 \pm 0.46$ & $100.0 \%$ & 1.00 \\
\hline & Total mean & $2.03 \pm 0.71$ & $94.8 \%$ & 0.89 \\
\hline
\end{tabular}

${ }^{\dagger}$ Anatomical detectability refers to a score of 1,2 , or 3 .

${ }^{\ddagger}$ Multi-rater kappa values between all three observers for each component.

\section{Image Quality}

The mean score for image artefacts was $1.01 \pm 0.65$ (Table 1). Because of the insensitivity of the used MRI method to susceptibility differences or off-resonance effects, remaining artefacts were only observed at abrupt jaw movements. Fig. (3B) presents selected images from a movie (Supportive Movie 2) of a subject with a transient though severe blurring of structures (arrows), which was caused by a very rapid movement (artifact score 2). The inter-observer agreement with respect to motion artifacts was moderate $(\kappa=$ 0.48 ) as one observer tended to generally rate for fewer artifacts than the other two. When the data from this observer were omitted, a substantial agreement was found between the other two observers with $\kappa=0.63$.

The mean score for anatomical detectability of major TMJ structures was $2.03 \pm 0.71$ (Table 2). Of all evaluated structures, more than $70 \%$ were rated as having good or excellent image quality (a score of 1 or 2) and over $95 \%$ were rated as positive for detection with diagnostic confidence (a score of 1, 2 or 3). For individual structures, almost $100 \%$ detectability was obtained for the condylar head independent of its position $(\geq 99.5 \%)$ and for all structures during movement $(\geq 97.3 \%)$. Inter-observer agreement for the rating of anatomical detectability was very $\operatorname{good}(\kappa=0.89)$.

\section{Joint Function}

With respect to disc displacement, 41 cases $(68.3 \%)$ were determined as normal, ten cases $(16.7 \%)$ as ADDR, and one case $(1.7 \%)$ as ADDNR. No posterior, lateral or medial displacements were observed. Eight cases (13.3\%) did not reach an agreement between normal and ADDR among three observers. For condyle movements, 34 cases (56.7\%) were determined as normal with continuous movement and 16 cases $(26.7 \%)$ as discontinuous movement. One case $(1.7 \%)$ did not reach an agreement for a movement with normal or limited range and ten cases (16.7\%) did not reach an agreement for a continuous or discontinuous movement. Nevertheless, there were very good inter-observer agreements for ratings of the disc displacement $(\kappa=0.91)$ and condyle movement $(\kappa=0.83)$.

Fig. (4) shows images from real-time MRI movies of a subject with anterior disc displacement (A) without reduction of the right TMJ and (B) with reduction of the left TMJ (Supportive Movies 3 and 4). The movements of the disc (arrows) and condyle as well as their relationship are clearly visualized. In particular, the time point of disc reduction is indicated by the abrupt movement of the condyle and the positional change of the disc (arrowhead).

\section{DISCUSSION}

In comparison to previous studies of TMJ dynamics [713], the present results are superior in terms of spatial resolution, tissue contrast, and residual artefacts. A brief review and comparison with the present work is summarized in Table 3. While susceptibility or off-resonance artefacts are completely avoided due to the very short echo times used here, the observation of transient motion artefacts is exclusively associated with the occurrence of abrupt joint movements. These problems must be attributed to the still relatively long acquisition time (1.66 s), which introduces data inconsistencies for objects with a sudden change of position. Rapid motions normally occur at the moment of disc recapture (as in the case of Fig. 3B) or when subjects feel a catch in their jaws (as in the case of Fig. 2B), which is caused by the internal derangement and often leads to joint "clicks and pops". However, because of the good visibility 


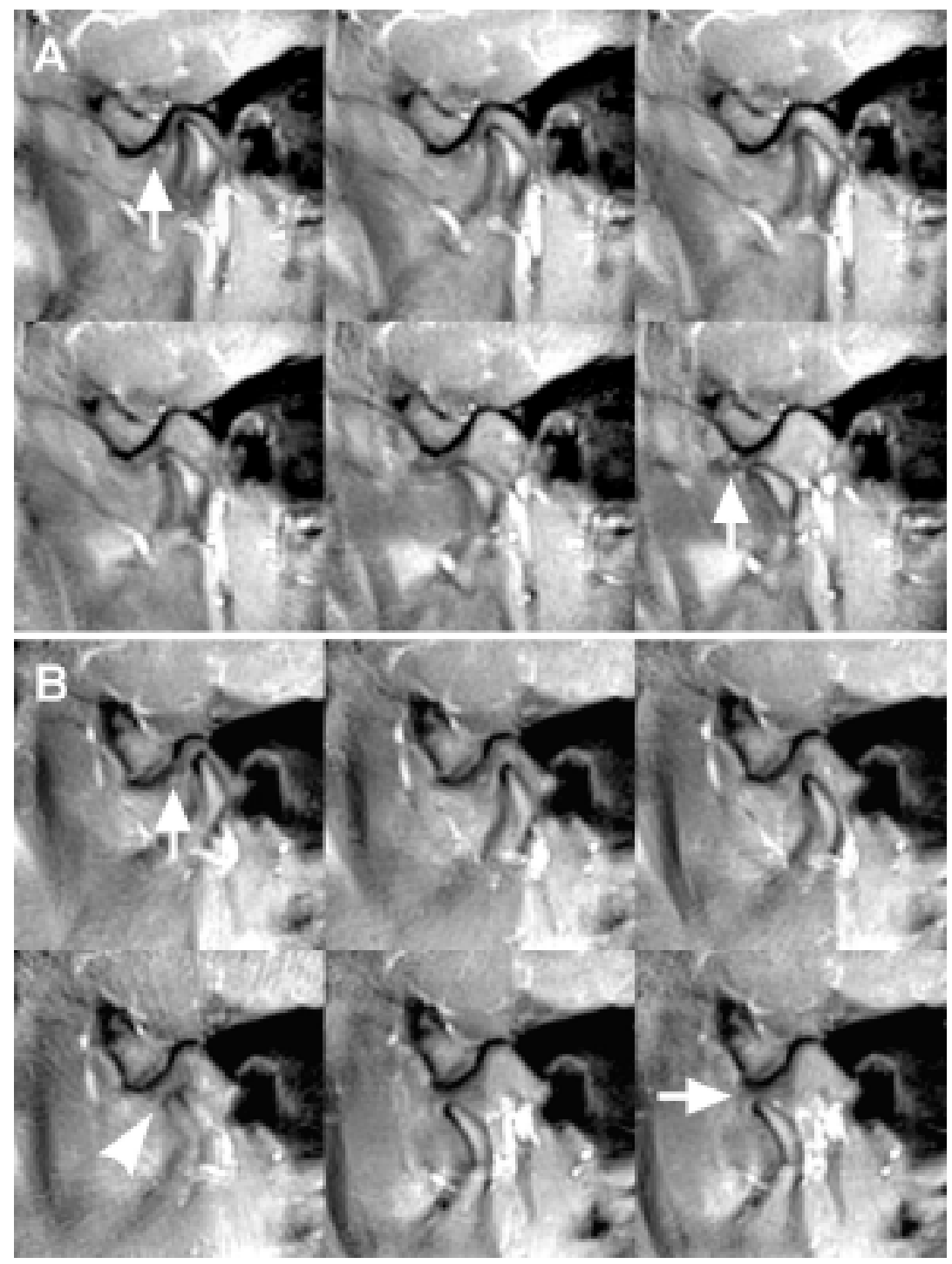

Fig. (4). Real-time MRI of abnormal TMJ function with anterior disc displacement without reduction (A) and anterior disc displacement with reduction (B). The images (parameters as in Fig. 3) were selected every $5 \mathrm{~s}$ (upper left to bottom right) from the corresponding movies (Supportive Movies 3 and 4) of the right and left TMJ of a 23-year-old female subject. The dislocated disc is indicated by arrows, while the moment of the disc recapture is indicated by an arrowhead.

of the disc-condyle relationship throughout the movement, transient artefacts did not preclude the evaluation of joint dynamics. In fact, the achieved image quality resulted in a high detectability of all major TMJ structures $(94.8 \%)$. In agreement with a recent study [11] slightly lower rates were found at the closed-mouth position for the anterior (84.9\%) and posterior borders $(79.0 \%)$ of the disc. The single case, where the disc body was hardly visible at all stages of the movement, led to a detection rate of $98.3 \%$ for the articular disc during movement, as opposed to $100 \%$ for the mandibular condyle (Table 2). The reason is believed to be an inappropriate biomechanical stress of the TMJ, which causes erosion of the condyle or temporal bone and results in a thin flat disc with possible perforation [18].

Despite advances in image quality, patient comfort (e.g., no bite block), and examination times (e.g., less than $8 \mathrm{~min}$ ), two limitations of the present study are the need for a relatively slow jaw movement and the restriction to a singleslice acquisition. Fortunately, it is foreseeable that a most recent innovation for real-time MRI that combines the present acquisition technique [14] with a nonlinear inverse image reconstruction [19] will solve this problem. The new MRI method allows for highly undersampled acquisitions of radial FLASH images and therefore leads to a further reduction of the acquisition time [20]. Preliminary trials yielded a 5-fold increase in temporal resolution for studies of TMJ movements without a sacrifice in image quality.

In conclusion, the present study reports on a robust visualization of the moving TMJ by real-time MRI with good image quality. The proposed method promises valuable information about the disc and condyle mobility, disc reduction, and topographic changes of the disc-condyle relationship at all stages of an active jaw movement. 
Table 3. Real-Time MRI Studies of TMJ Dynamics

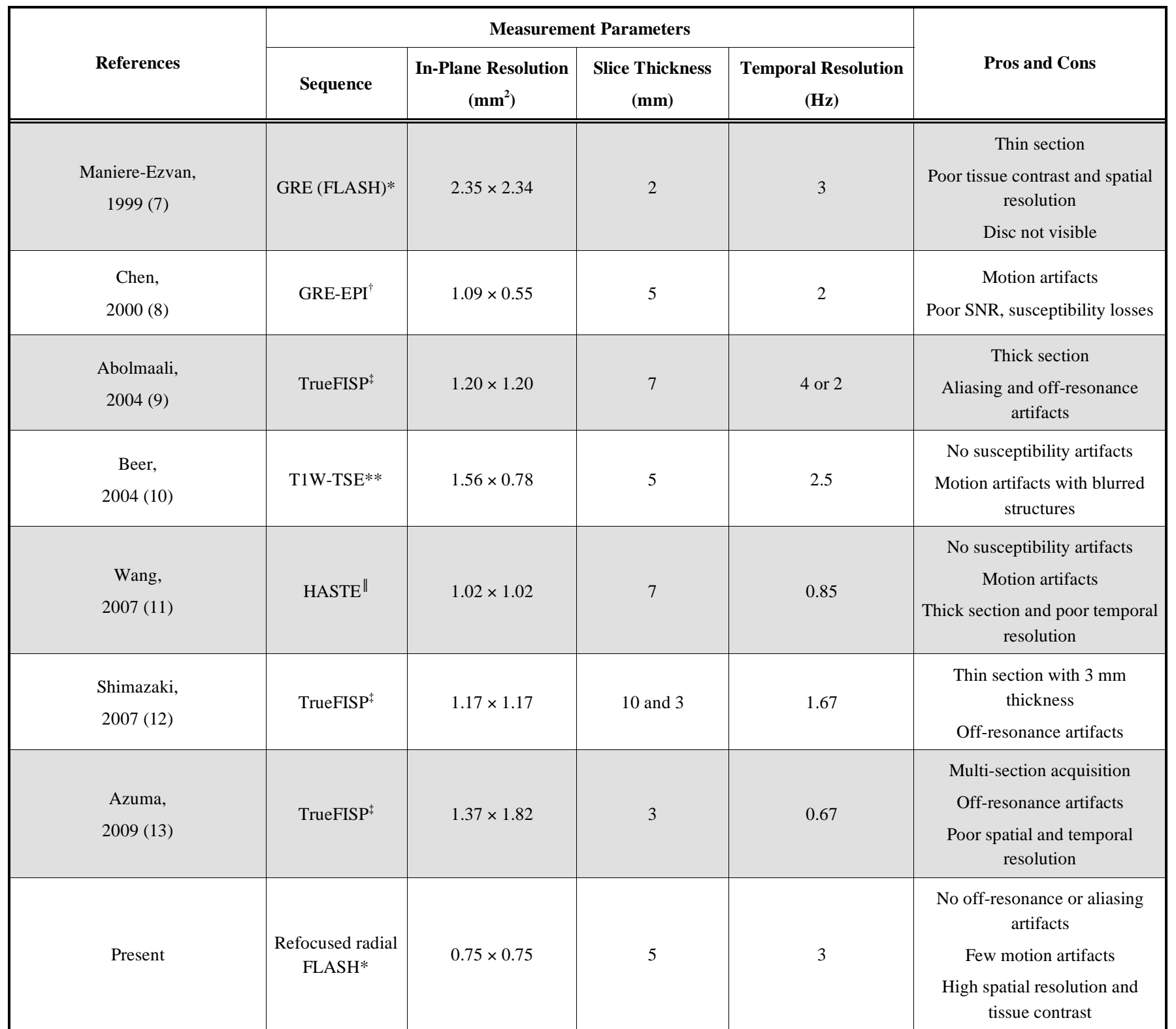

*GRE: gradient echo / FLASH: fast low angle shot; ${ }^{\dagger}$ EPI: echo planar imaging; ${ }^{\ddagger}$ TrueFISP: true fast imaging with steady-state precession; ** T1W-TSE: T1-weighted turbo spin echo; "HASTE: half-Fourier acquisition single shot turbo spin echo

Thorough patient studies are warranted to assess the diagnostic sensitivity, specificity and accuracy.

\section{ACKNOWLEDGEMENTS}

The authors thank the late Prof. Dr. Alfons Huels, Dr. Klaus-Dietmar Merboldt, and Dr. Mathias Wasser for image evaluation and Olaf Kling for help in the review of the literature.

\section{SUPPLEMENTARY MATERIAL}

Supplementary material is available on the publishers Web site along with the published article.

\section{REFERENCES}

[1] Marguelles-Bonnet RE, Carpentier P, Yung JP, Defrennes D, Pharaboz C. Clinical diagnosis compared with findings of magnetic resonance imaging in 242 patients with internal derangement of the TMJ. J Orofac Pain 1995; 9: 244-53.

[2] Emshoff R, Brandlmaier I, Bertram S, Rudisch A. Comparing methods for diagnosing temporomandibular joint disk displacement without reduction. J Am Dent Assoc 2002; 133: 442-51.

[3] Burnett KA, Davis CL, Read J. Dynamic display of the temporomandibular joint meniscus by using "fast-scan" MR imaging. Am J Roentgenol 1987; 149: 959-62.

[4] Schellhas KP, Fritts HM, Heithoff KB, Jahn JA, Wilkes CH, Omlie MR. Temporomandibular joint: MR fast scanning. Cranio 1988; 6 : 209-16.

[5] Conway WF, Hayes CW, Campbell RL. Dynamic magnetic resonance imaging of the temporomandibular joint using FLASH sequences. J Oral Maxillofac Surg 1988; 46: 930-8.

[6] Behr M, Held P, Leibrock A, Fellner C, Handel G. Diagnostic potential of pseudo-dynamic MRI (CINE mode) for evaluation of internal derangement of the TMJ. Eur J Radiol 1996; 23: 212-5.

[7] Maniere-Ezvan A, Havet T, Franconi JM, Quemar JC, de Certaines JD. Cinematic study of temporomandibular joint motion using ultra-fast magnetic resonance imaging. Cranio 1999; 17: 262-7. 
[8] Chen YY, Callo LM, Meier D, Palla S. Dynamic Magnetic resonance imaging technique for the study of the temporomandibular joint. J Orofac Pain 2000; 14: 65-73.

[9] Abolmaali ND, Schmitt J, Schwarz W, Toll DE, Hinterwimmer S, Vogl TJ. Visualization of the articular disk of the temporomandibular joint in near-real-time MRI: feasibility study. Eur Radiol 2004; 14: 1889-94.

[10] Beer A, Kolk A, Neff A, Hof N, Treumann T, Rummeny EJ. CineMRT des Kiefergelenks im Vergleich zur konventionellen MRT und Achsiographie. RoFo 2004; 176: 506-12.

[11] Wang EY, Mulholland TP, Pramanik BP, et al. Dynamic sagittal half-Fourier acquired single-shot turbo spin-echo MR imaging of the temporomandibular joint: initial experience and comparison with sagittal oblique proton-attenuation images. Am J Neuroradiol 2007; 28: 1126-32.

[12] Shimazaki Y, Saito K, Matsukawa S, et al. Image quality using dynamic MR imaging of the temporomandibular joint with trueFISP sequence. Magn Reson Med Sci 2007; 6: 15-20.

[13] Azuma T, Ito J, Kutsuki M, Nakai R, Fujita S, Tsutsumi S. Analysis of the mandibular movement by simultaneous multisection continuous ultrafast MRI. Magn Reson Imaging 2009; 27: 423-33.
[14] Zhang S, Block KT, Frahm J. Magnetic resonance imaging in real time: Advances using radial FLASH. J Magn Reson Imaging 2010; 31: 101-9.

[15] Dworkin SF, Leresche L. Research diagnostic criteria for temporomandibular disorders: review, criteria, examinations, and specifications, critique. J Craniomand Disord 1992; 120: 273-81.

[16] Orsini MG, Kuboki T, Terada S, Matsuka Y, Yamashita A, Clark GT. Diagnostic value of 4 criteria to interpret temporomandibular joint normal disk position on magnetic resonance images. Oral Surg Oral Med O 1998; 86: 489-97.

[17] Randolph JJ. Free-marginal multirater kappa: An alternative to Fleiss' fixed-marginal multirater kappa. Joensuu University Learning and Instruction Symposium, Oct 14-15, 2005, Joensuu, Finland. doi: 10.1.1.59.8776.

[18] Beek M, Aarnts MP, Koolstra JH, Feilzer AJ, van Eijden TM. Dynamic properties of the human temporomandibular joint disc. J Dent Res 2001; 80: 876-80.

[19] Uecker M, Hohage T, Block KT, Frahm J. Image reconstruction by regularized nonlinear inversion - Joint estimation of coil sensitivities and image content. Magn Reson Med 2008; 60: 67482.

[20] Uecker M, Zhang S, Voit D, Karaus A, Merboldt KD, Frahm J. Real-time magnetic resonance imaging at $20 \mathrm{~ms}$ resolution. NMR Biomed 2010; 23: 1-8. 